\title{
Tissue Plasminogen Activator for the Treatment of Parapneumonic Effusions in Pediatric Patients
}

\author{
Emily N. Israel $^{1,2}$ and Allison B. Blackmer ${ }^{1,2, *}$ \\ ${ }^{1}$ Department of Pharmacy Services, University of Michigan Health System, Ann Arbor, Michigan; ${ }^{2}$ Department of \\ Clinical, Social, and Administrative Sciences, University of Michigan College of Pharmacy, Ann Arbor, Michigan
}

Intrapleural fibrinolysis has been investigated for the treatment of pleural effusion for several decades. Fibrinolytics have the ability to break up fibrin and loculations that characterize complicated pleural effusions, facilitating drainage. Older fibrinolytics such as urokinase and streptokinase have been replaced by tissue plasminogen activator (tPA) for this indication due to product availability and a more favorable safety profile. The literature supports tPA as a treatment approach for this indication in adult patients, and the use of tPA has become a standard management approach in this population. Over the past decade, data on the efficacy of intrapleural fibrinolytic therapy in children have also been generated, which now support the use of fibrinolysis as a treatment alternative to more invasive therapeutic options such as surgical intervention. In this review, we discuss the pathophysiology, diagnosis, and treatment of parapneumonic effusion and empyema, with a focus on intrapleural fibrinolysis, specifically tissue plasminogen activator, in the pediatric population. Recent articles provide sufficient evidence to support the use of this drug in pediatric patients for the management of pleural effusions; however, due to study heterogeneity, questions remain that may be addressed in future studies.

KeY Words pediatric, tissue plasminogen activator, pleural effusion, empyema, fibrinolytic, parapneumonic effusion.

(Pharmacotherapy 2014;34(5):521-532) doi: 10.1002/phar.1392

Pleural effusion is defined as the accumulation of excess fluid in the pleural space between the lung and the chest wall. ${ }^{1}$ Several possible etiologies for pleural effusion are heart failure, trauma, liver disease, and malignancy, as well as chylous and transudative effusions. ${ }^{2}$ However, in the pediatric population, pleural effusions are most commonly caused by infection-typically a bacterial pneumonia - and are thus termed parapneumonic effusions. ${ }^{3}$ Empyemas are more advanced, organized effusions and refer to loculations or pus in the pleural space. ${ }^{1}$ Although not all effusions require intervention, those that

*Address for correspondence: Allison B. Blackmer, Department of Pediatric Surgery, College of Pharmacy, C.S. Mott Children's Hospital, The University of Michigan, 1540 East Hospital Drive, Room 10-561B, Ann Arbor, MI 48109; e-mail: ablackm@umich.edu.

(C) 2014 Pharmacotherapy Publications, Inc. are infectious in origin, are more advanced, or large enough in volume to affect respiratory dynamics may necessitate medical and/or surgical treatment. ${ }^{4-6}$

After the introduction of routine immunization of infants with the 7-valent pneumococcal conjugate vaccine in 2000, the hospitalization rate associated with all-cause pneumonia in children younger than 2 years of age declined by $33 \%$ in the United States. ${ }^{7}$ Despite this decrease, the rate of empyema complicating communityacquired pneumonia in children has risen across that same time period; specifically, the reported rate was 3.5 cases $/ 100,000$ children from 1996 1998 , whereas the rate was 7 cases/100,000 children from 2005-2007. ${ }^{7}$ Pleural effusion may complicate up to $60 \%$ of pneumonias. ${ }^{8-10}$ Symptoms are related to the size and extent of the pleural effusion and may include fever, tachypnea, and increased oxygen requirements. 
Effusions may subsequently progress to empyemas, which increase the risk of morbidity and mortality. Mortality associated with empyema is roughly $20 \%$ in adults but is significantly lower in children. ${ }^{4,9}$ However, empyema in the pediatric population is still associated with significant morbidity including restrictive lung disease and the necessity for advanced medical and invasive surgical interventions. ${ }^{4}$

Intrapleural fibrinolysis was initially investigated nearly 60 years ago for the treatment of empyema and has now become a standard management approach in adult patients. ${ }^{10,11}$ Over the past decade, data on the efficacy of fibrinolytic therapy in children have been generated, which now support fibrinolysis as a treatment alternative to invasive surgical interventions. In this review, we discuss the pathophysiology, diagnosis, and treatment of parapneumonic effusion and empyema, with a focus on intrapleural fibrinolysis with tissue plasminogen activator (tPA), in the pediatric population.

\section{Pathophysiology}

Parapneumonic effusions and empyemas are commonly caused by the typical pathogens responsible for bacterial pneumonia: Streptococcus pneumoniae, Streptococcus pyogenes, Staphylococcus aureus, Haemophilus influenzae, Pseudomonas aeruginosa, and Mycoplasma pneumoniae. 3, 4, 12, 13 In response to infection, pleural secretions and capillary permeability are increased, resulting in an accumulation of fluid. This fluid collectionthe pleural effusion-can cause a mass effect, resulting in restriction of lung expansion and eventual increased oxygen requirements. ${ }^{11,} 12$ The stimulation of the inflammatory cascade may also lead to subsequent activation of the coagulation cascade and fibrin deposition, ultimately leading to the formation of an empyema. ${ }^{11,12}$ The disease process develops along a time continuum through three stages: exudative, fibrinopurulent, and organizational. The risk of poor outcome increases as the disease progresses. The presence of pus is typically associated with the worst outcomes; however, any of the criteria associated with moderate- or high-risk effusions confer a risk of poor outcome..$^{5}$

The exudative stage is characterized by a rapid accumulation of free-flowing fluid in the pleural space. This is secondary to interstitial fluid crossing the pleura and entering the pleural space due to inflammation and increased capillary permeability. ${ }^{1,12}$ At this point, the effusion is still considered to be uncomplicated. However, the fluid frequently becomes secondarily infected and marks the transition of the effusion into the fibrinopurulent stage. The fluid collection at this stage becomes increasingly complicated as the infection leads to continued inflammation. ${ }^{12}$ Increased metabolic activity due to microbial growth affects the chemistry of the pleural fluid and causes the glucose concentration and $\mathrm{pH}$ to drop. $^{7}$ Neutrophil lysis also causes a concurrent rise in the lactate dehydrogenase concentration. ${ }^{7}$ In this phase, the coagulation cascade and fibrinolysis become imbalanced, leading to fibrin deposition, loculations, and adhesions. ${ }^{12}$ In the third phase of disease progression, the organizational phase, the fluid collection develops into an empyema. As advanced complicated effusions become organized, pus develops within the pleural space, and fibroblasts produce a thick, fibrous pleural peel. ${ }^{12}$ Loculations develop further, and the thickened collection creates a mass effect, preventing effective lung expansion. ${ }^{12}$ Table 1 depicts the physiologic characteristics of each of these three stages.

\section{Diagnosis}

Multiple diagnostic modalities are available for evaluating pleural effusion in pediatric patients. The main imaging options include chest radiograph (CXR), ultrasound, and computed tomography (CT). ${ }^{9}$ Initial CXR is often used to identify a possible effusion; however, it cannot specifically differentiate between freeflowing effusion and loculated empyema, or even pneumonia-related lung consolidation. ${ }^{9}, 14$ Serial CXRs may be particularly helpful when evaluating disease progression and the effect of therapy in children who are not clinically improving with standard care. ${ }^{3,13}$ In addition, CXR can help identify other conditions associated with parapneumonic effusion and empyema such as pneumothorax, lobar consolidation, air fluid levels, and pneumatoceoles. ${ }^{14}$

The American Pediatric Surgical Association (APSA) Outcomes and Clinical Trials Committee published recommendations in 2012 for the diagnosis and management of empyema in children. The APSA recommends ultrasound as the first-line option for imaging in children with suspected pleural effusion based on CXR. ${ }^{9}$ Ultrasound has many advantages in this population including low relative cost, portability, and lack of associated radiation. However, the utility of ultrasound is dependent on the quality of images 
Table 1. Phases of Pleural Effusion ${ }^{1,}$, 12

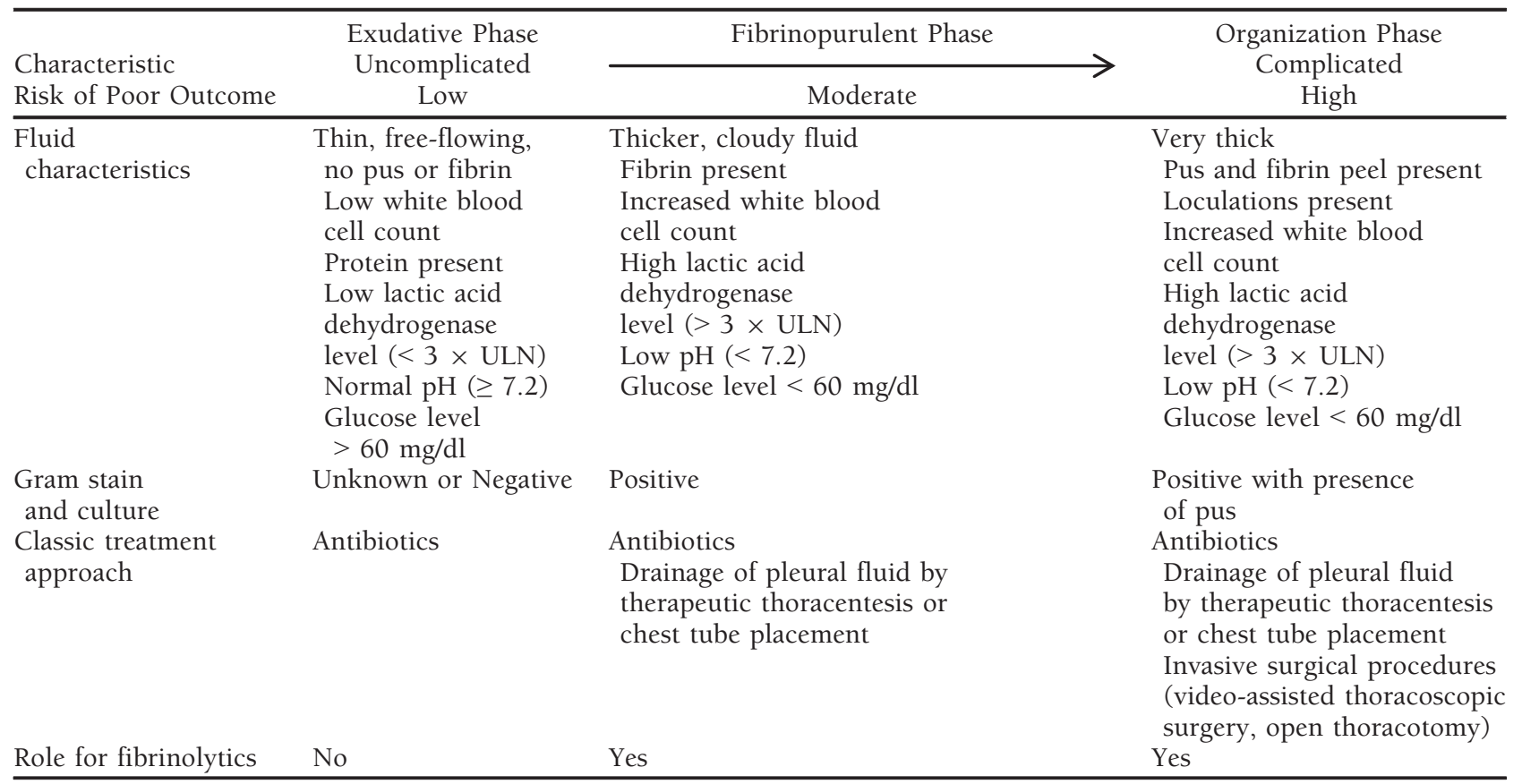

ULN $=$ upper limit of normal.

and operator expertise. Further, ultrasound may not be readily available at all institutions. Computed tomography offers an alternative diagnostic modality. Similar to ultrasound, CT provides information about pleural thickening and loculations; however, it may be less accurate than ultrasound in identifying fibrin deposits. Computed tomography is significantly more expensive than ultrasound. Additionally, radiation exposure associated with routine $\mathrm{CT}$ use may increase the long-term risk of malignancy. The APSA recommends using CT only in complex cases in which ultrasound is inadequate or additional information is required for surgical decision making.

Definitive diagnosis is possible with diagnostic thoracentesis, a procedure involving the aspiration of pleural fluid from the effusion with a needle. ${ }^{10}$ The collected fluid is analyzed for characteristic diagnostic findings across the stages of disease (Table 1).

\section{Treatment}

A summary of the classic treatment approaches across the stages of disease is included in Table 1. Exudative (or simple) parapneumonic effusions are often effectively managed with medical therapy alone. Early initiation of appropriate antibiotics can decrease the severity and duration of effusions. ${ }^{3}$ Empiric ther- apy should be selected based on the suspicion for community- versus hospital-acquired infections, the patient's immunization status, as well as local resistance patterns and suspicion for more virulent organisms such as community acquired methicillin-resistant Staphylococcus aureus. $3,4,10,13$ The pleural fluid may also be cultured, obtained either by thoracentesis or drainage with tube thoracostomy, in order to help further guide and tailor antibiotic therapy. ${ }^{3}$

In patients with more advanced (or complex) pleural effusions, antibiotic therapy alone may be insufficient, and additional drainage of the pleural fluid is necessitated. ${ }^{3}$ Initial options for drainage of pleural fluid include therapeutic thoracentesis or chest tube placement as monotherapy, or chest tube placement with concomitant chemical debridement (e.g., fibrinolytic therapy). ${ }^{3,}{ }^{4}$ First-line therapy often includes chest tube drainage; but, therapeutic thoracentesis is somewhat less invasive and may be pursued initially. ${ }^{12}$ If thoracentesis is used as first-line therapy, the 2011 British Thoracic Society publication recommends placement of a chest tube after the first failed thoracentesis attempt (e.g., fluid reaccumulation). ${ }^{3}$ However, drainage by repeated thoracentesis has not been compared with chest tube placement alone.

Drainage may be unachievable at more advanced, or complex, stages of pleural effusion due to fibrin deposition and loculations. ${ }^{8}$ In 
patients unable to achieve sufficient drainage of pleural fluid, additional intervention is warranted in order to avoid continued lung restriction, increased oxygen requirements, and progression to higher levels of respiratory support such as mechanical ventilation or extracorporeal membrane oxygenation. ${ }^{3}$ The historical gold standard of care for more progressive empyema is surgical intervention. The two most common surgical procedures for progressive empyema include minimally invasive videoassisted thoracoscopic surgery (VATS) and open thoracotomy for decortication of loculations. ${ }^{5}$

The VATS procedure is currently considered the first-line surgical option for qualifying patients. It is a newer, highly effective technique that allows for debridement and decortication of loculations through minimally invasive access and direct visual inspection of the pleural space with the use of a laprascope. ${ }^{3}$ As a minimally invasive surgery, patients may experience less postoperative pain and a quicker recovery period compared with other surgical options. Furthermore, the risk of long-term associated morbidity is lessened. However, some patients are not candidates for this procedure and/or may eventually require a more invasive approach. Open thoracotomy with decortication, although extremely effective and thorough, remains the most invasive option for debridement and may be required in up to $35 \%$ of patients who fail conservative medical measures or in those who fail the VATS procedure. ${ }^{8}$ Compared with VATS, the open thoracotomy procedure requires a larger surgical incision, is highly invasive, and is associated with significantly more postoperative pain, a longer recovery period, and additional postoperative morbidity (e.g., potential for rib fusion and scoliosis). Both VATS and open thoracotomy are associated with the risk of postsurgical complications such as bleeding and the requirement for extended ventilator support.

\section{Fibrinolytic Pharmacology and Pharmacokinetics}

Since the early 1950s, fibrinolytic therapy has emerged as a less invasive alternative treatment approach to the more invasive surgical procedures for complicated effusions described above. The theory behind the use of fibrinolytics is rooted in the idea that complicated parapneumonic effusions and empyemas are characterized by fibrin deposition. ${ }^{8}$ The ability to break up fibrin and loculations with intrapleural fibrinolytics allows for greater success in achieving drainage without further surgical or mechanical interventions.

Although not currently available in the United States, the first-generation fibrinolytics, streptokinase and urokinase, were initially used to treat parapneumonic effusions and empyema. ${ }^{15-18}$ Streptokinase indirectly activates the fibrinolytic system by binding plasminogen and causing conformational change. ${ }^{12,19}$ An active site is subsequently exposed and cleaves other plasminogen molecules to promote the formation of plasmin. ${ }^{19}$ Urokinase, a thrombolytic agent obtained from human neonatal kidney cells grown in culture, works slightly differently; one plasmin molecule can be directly activated by each urokinase molecule. ${ }^{12,20}$ Plasmin then promotes the breakdown of fibrin. ${ }^{19}$ Although more common with streptokinase, both agents have been associated with adverse effects, specifically, immunologic and hypersensitivity reactions. ${ }^{12}$ Furthermore, an antibody-mediated response that caused ineffectiveness with repeat dosing was also associated with streptokinase use because its production is bacteria based. ${ }^{12,17}$

Tissue plasminogen activator was developed as an answer to some of the drawbacks associated with the first-generation agents. It binds directly to fibrin and catalyzes the conversion of plasminogen to plasmin, leading to fibrinolysis. ${ }^{12}$ Furthermore, tPA is not associated with hypersensitivity reactions and retains its effectiveness over multiple doses. ${ }^{12}$ Although the pharmacokinetic parameters of intrapleural tPA have not been definitively determined, when given intravenously, the volume of distribution approximates plasma volume. Clearance is rapid from the plasma through metabolism in the liver, and the half-life of tPA is less than 5 minutes in adult patients. ${ }^{21}$

Approved indications for systemic tPA include management of acute myocardial infarction, acute ischemic stroke within 3 hours of stroke symptom onset, and acute massive or unstable pulmonary embolism in adults. ${ }^{21}$ Tissue plasminogen activator is also approved for restoring patency to central venous access devices. ${ }^{22}$ Unapproved indications include treatment of arterial thrombosis and fistula thrombosis, ocular surgery, vascular graft occlusion, venous thromboembolism, and intrapleural use. ${ }^{23}$

Absolute contraindications to systemic tPA therapy include previous allergic reaction, trauma within the previous 48 hours, or bron- 
chopleural fistula. ${ }^{12,} 19$ Relative contraindications include history of hemorrhagic stroke; coagulation deficiencies; and major thoracic, abdominal, or cranial surgery within the previous 2 weeks. ${ }^{12,19}$ Contraindications to intrapleural administration of tPA are not well established and are founded on clinical experience and safety risks published in the literature.

\section{Supporting Literature in Adults}

The use of fibrinolytic therapy is well established in the literature in adults, with numerous case reports, retrospective reviews, and randomized trials supporting its use for the treatment of complicated pleural effusions and empyemas. $^{24-30}$ A comprehensive review of the adult literature was provided in a 2010 publication. ${ }^{12}$ Intrapleural tPA has demonstrated improved chest tube drainage, resolution of symptoms, decreased need for invasive surgical procedures, and shorter hospital stays. ${ }^{12}$ The literature supporting the use of intrapleural tPA in adults, however, is heterogeneous with regard to indications, therapeutic end points, dosing, frequency, duration of treatment, and dwell time.

A recent investigation in the adult population evaluated dual intrapleural therapy with tPA plus recombinant human dornase alfa. ${ }^{29}$ Empyema fluid typically contains large amounts of extracellular DNA. Dornase alfa (or DNase) degrades polymerized deoxyribonucleoproteins in bacterial and mammalian cells, theoretically reducing pus viscosity and facilitating drainage. $^{12,29}$ In a multicenter, prospective trial, 210 adults with parapneumonic effusions were randomized to receive one of four possible treatments: tPA plus dornase alfa, dornase alfa plus placebo, tPA plus placebo, or double placebo. ${ }^{29}$ Dornase alfa $5 \mathrm{mg}$ plus tPA $10 \mathrm{mg}$ were given twice/day for 3 days, with a dwell time of 1 hour. The group receiving combination therapy with dornase alfa and tPA showed a significant decrease in the area of pleural opacity on CXR. Patients who received dual therapy were also less likely to require surgical referral, had increased pleural fluid drainage, and had decreased total length of hospital stay. The other three treatment arms did not show a statistically significant benefit. No significant differences in adverse effects among the groups were reported. Thus, dual therapy with intrapleural tPA and dornase alfa is concluded to be safe and effective in adults with parapneumonic effusion.

\section{Supporting Literature in Pediatric Patients}

Since the 1990s, investigations of fibrinolytic therapy have expanded into the pediatric population. The efficacy of intrapleural fibrinolytics in the pediatric population has been demonstrated in several retrospective studies, case studies, and case series (Table 2), as well as one prospective study.

Retrospective Data

\section{Early Use of tPA in Pediatric Patients}

Early case reports and case series described successful treatment with tPA in pediatric patients as demonstrated by resolution of clinical signs and symptoms, increased chest tube drainage, improvements seen on CXR and CT scan, and prevention of surgical intervention. ${ }^{8,} 31$ The first published case of successful intrapleural tPA in a pediatric patient, published in 2001, suggested preliminary efficacy in the pediatric population. ${ }^{8}$ A 6-year-old boy received tPA $5 \mathrm{mg}$ diluted in $50 \mathrm{ml}$ of normal saline by chest tube instillation for management of pleural effusion. The chest tube was clamped for 1 hour, and a second dose was given 8 hours later. Chest tube drainage was increased, the CXR demonstrated improvement, and invasive, surgical interventions were avoided.

Similar findings were published in $2003 .{ }^{31} \mathrm{~A}$ 10.3-kg, 16-month-old female received tPA $2 \mathrm{mg}$ directly instilled intrapleurally for a persistent parapneumonic effusion, decreased chest tube drainage, and ongoing clinical signs and symptoms despite appropriate antibiotic therapy. Over 6 days, the patient received four doses of tPA, each dwelling for a 4-hour period. Treatment success was measured by increased chest tube drainage, improvement of clinical signs and symptoms, and resolution seen on both CT scan and CXR.

A small case series of six children aged 2-13 years old evaluated the efficacy of tPA as measured by the difference in chest tube output before and after tPA administration. ${ }^{32}$ Patients received tPA $2-5 \mathrm{mg}$ mixed in normal saline with a dwell time ranging from 4-6 hours. Doses were repeated every 12-24 hours until resolution was observed on CXR or therapy was no longer deemed to be efficacious (e.g., volume instilled equaled volume drained). Patients received, on average, a total of 3-6 doses. In addition, unlike any other published report, patients were rotated 


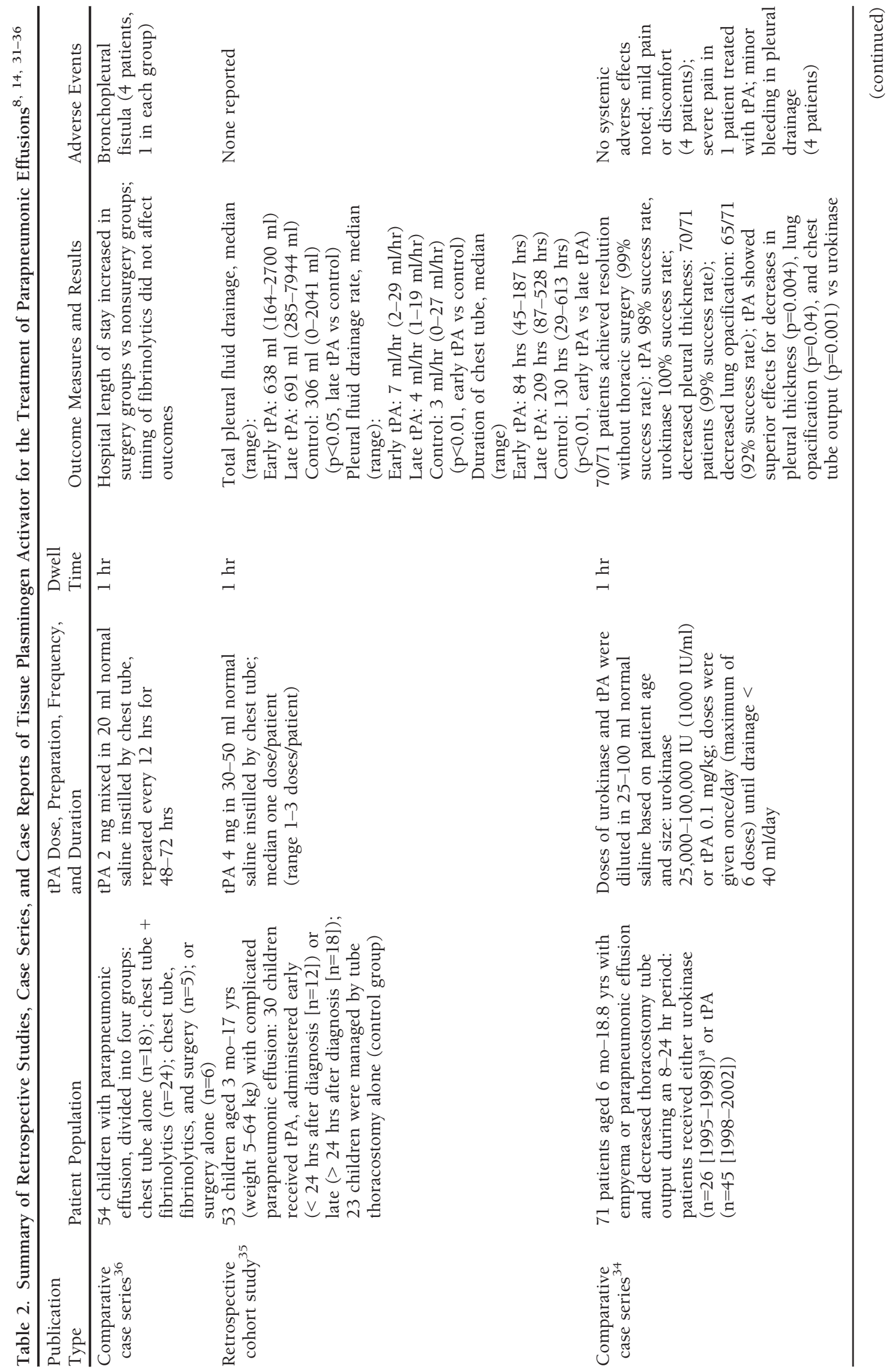




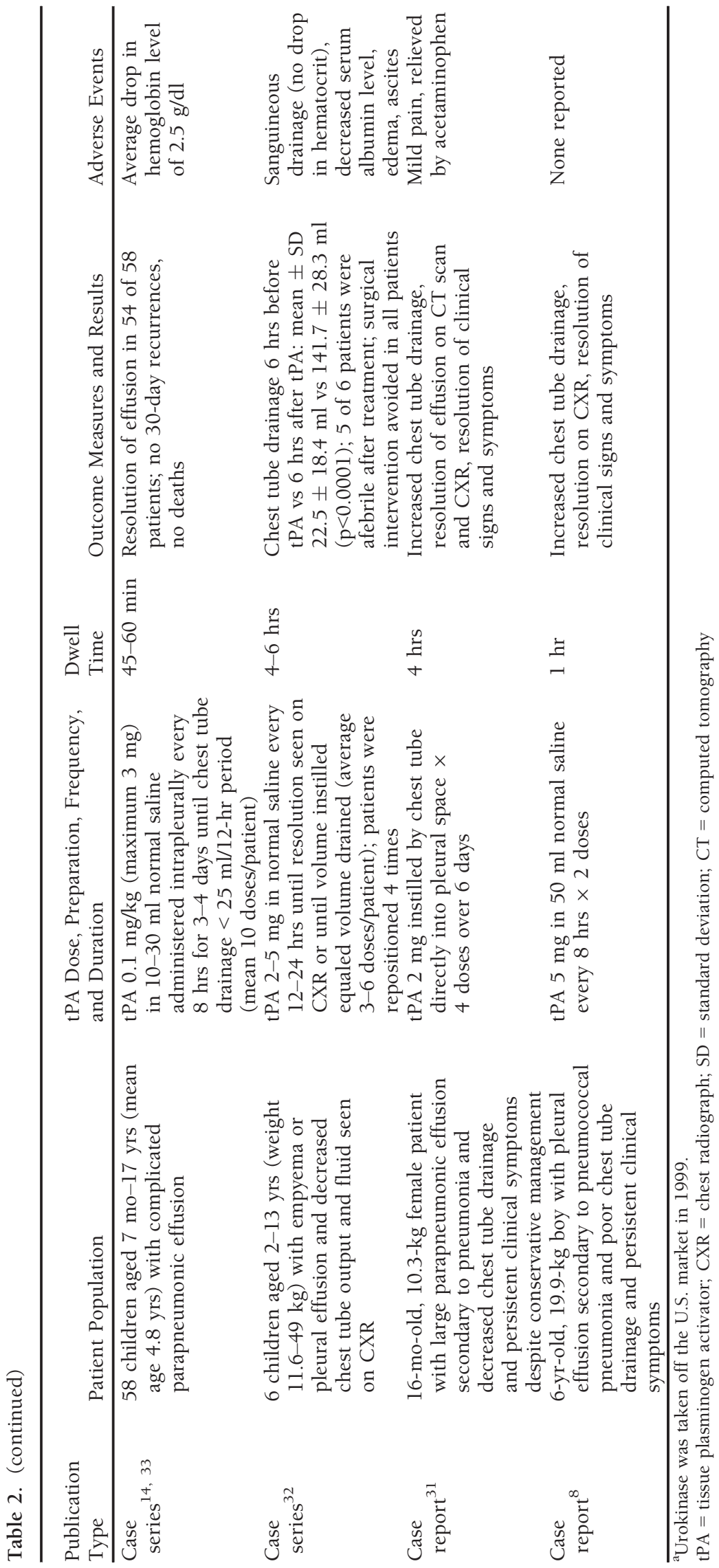


at 30-minute intervals into the Trendelenburg, reverse Trendelenburg, and left and right lateral decubitus positions. The primary end point of the difference in pleural fluid drainage before tPA (mean \pm SD $22.5 \pm 18.4 \mathrm{ml}$ ) versus after tPA $(141.7 \pm 28.3 \mathrm{ml})$ administration was significant $(p<0.0001)$. Further, five of the six patients achieved effusion resolution.

A larger retrospective case series of 58 children with complicated parapneumonic effusion, confirmed results of earlier case reports. ${ }^{33}$ Children aged 7 months-17 years were treated with weight-based dosing of tPA: $0.1 \mathrm{mg} / \mathrm{kg} / \mathrm{dose}$ mixed in 10-30 $\mathrm{ml}$ of normal saline (maximum dose of $3 \mathrm{mg}$ ). The dwell time ranged from 4560 minutes, repeated every 8 hours for 34 days, until chest tube drainage was less than $25 \mathrm{ml} / 12$-hour period. The mean number of doses/patient was 10 . Of the 58 patients evaluated, 54 achieved resolution, and there were no 30-day recurrences and no deaths reported. Of the four patients who failed tPA therapy, three underwent the VATS procedure, and one patient had open thoracotomy with decortication and debridement. In a second large case series, 71 children, aged 6 months-18.8 years, with empyema or parapneumonic effusion and decreased chest tube output and pleural thickening seen on CXR were evaluated. ${ }^{34}$ This retrospective case series was completed between 1995 and 2002. As urokinase was still on the U.S. market from 1995-1998, patients received either urokinase 25,000-100,000 IU (26 patients) or tPA $0.1 \mathrm{mg} / \mathrm{kg}$ diluted in $25-100 \mathrm{ml}$ of normal saline (45 patients), depending on patient age and size. Doses were administered once/day and repeated each day until chest tube output was less than $40 \mathrm{ml} /$ day, with a maximum of six doses. Efficacy was demonstrated in both groups, with 70 of 71 patients achieving resolution of effusion without the need for invasive surgical intervention (99\% success rate). Similarly, a decrease in pleural thickness was observed in 70 of 71 patients and a decrease in lung opacifications was observed in 65 of 71 patients (92\% success rate). When compared with urokinase, tPA showed superior effects for decrease in pleural thickness $\quad(\mathrm{p}=0.004)$, lung opacification $(p=0.04)$, and chest tube output $(p=0.001)$.

\section{Timing of tPA Initiation}

A large, retrospective cohort study evaluated 53 children with complicated pleural effusion: 30 children were treated with tPA, and 23 chil- dren were managed by tube thoracostomy alone (control group). ${ }^{35}$ The study further evaluated the impact of the timing of tPA initiation by subdividing the treatment group into two groups: early tPA treatment $(<24$ hrs after diagnosis [12 patients]) and late tPA treatment (> 24 hrs after diagnosis [18 patients]). Patients ranged in age from 3 months-17 years old. Intrapleural tPA was dosed at 4 mg diluted in 30$50 \mathrm{ml}$ of normal saline and allowed to dwell for 1 hour; the median number of doses/patient was one (range of 1-3 doses/patient). The total median pleural fluid drainage was $691 \mathrm{ml}$ (range 285-7944 ml) in the late tPA group versus $360 \mathrm{ml}$ (range 0-2041) in the control group $(\mathrm{p}<0.05)$; however, a significant difference was not demonstrated between the early versus late groups. The difference in the rate of pleural fluid drainage between the early tPA group ( $7 \mathrm{ml} / \mathrm{hr}$ [range $2-29 \mathrm{ml} / \mathrm{hr}$ ]) versus the control group ( $3 \mathrm{ml} / \mathrm{hr}$ [range $0-27 \mathrm{ml} / \mathrm{hr}$ ], $\mathrm{p}<0.01$ ) was significant, and the difference in the duration of chest tube placement between the early tPA group ( 84 hrs [range 45-187 hrs]) versus the late group (209 hrs [range 87-528 hrs], p<0.01) was significant. No surgical interventions were required for those patients treated with tPA.

\section{Comparison of Treatment Options}

The efficacy of tPA for parapneumonic effusion and the timing of tPA initiation were described above; however, questions remain regarding the comparative effects of tPA versus other treatment options. A retrospective study evaluated 54 children with pleural effusion to make this comparison. ${ }^{36}$ In this study, patients were divided into four groups: chest tube alone (18 patients); chest tube with fibrinolytics (24 patients); chest tube, fibrinolytics, and surgery (5 patients); and surgery alone (6 patients). Children treated with intrapleural tPA were given $2 \mathrm{mg}$ diluted in $20 \mathrm{ml}$ of saline every 12 hours for 48-72 hours, with a dwell time of 1 hour. Outcome variables included hospital length of stay, duration of intensive care unit stay, duration of leukocytosis, days from ultimate therapy (i.e., the number of days from the last required therapy [e.g., chest tube monotherapy, chest tube plus fibrinolytic, or surgery] until discharge), and average hospital charges. Patients who received surgery had a longer hospital length of stay $(\mathrm{p}=0.002)$, longer intensive care unit stay $(\mathrm{p}=0.01)$, and more days from therapy to discharge $(p=0.044)$. In addition, 
hospital charges were lower in those patients who did not receive surgery.

\section{Prospective Data}

To our knowledge, only one prospective, randomized trial has evaluated intrapleural tPA in pediatric patients. ${ }^{37}$ In this trial, fibrinolysis with tPA was compared with VATS as initial treatment in pediatric patients ( $<18$ yrs of age) with empyema. Thirty-six patients with empyema diagnosed by CT or ultrasound or who had purulent drainage with a white blood cell count above $10 \times 10^{3} / \mu \mathrm{l}$ were randomly assigned to undergo VATS $(n=18)$ or to receive intrapleural tPA therapy (18 patients). All patients were treated with similar and appropriate antibiotic regimens. Patients were excluded if they had contraindications to thoracoscopy or fibrinolysis, any additional foci of infection, immunocompromised state, or comorbid conditions requiring hospitalization beyond empyema treatment. Children who received tPA had 12-French chest tubes in place. At the time of chest tube placement, tPA $4 \mathrm{mg}$ mixed in $40 \mathrm{ml}$ of normal saline was instilled directly into the tube, with a dwell time of 1 hour. Treatment was repeated every 24 hours for a total of three doses. The primary outcome measures were hospital length of stay, posttherapy days of oxygen therapy, days until afebrile, number of analgesic doses, requirements for a second procedure, and total hospital cost.

Baseline demographic data were well matched in each group. Organisms cultured from both groups were consistent with the typical pathogens associated with pleural effusion. Outcome measures were similar in the VATS versus fibrinolysis groups for hospital length of stay after intervention (mean \pm SD $6.9 \pm 3.7 \quad$ vs $6.8 \pm 2.9$ days, $\mathrm{p}=0.96)$, posttherapy days of oxygen $(2.3 \pm 1.7$ vs $2.3 \pm 2.1$ days, $p=0.9)$, days until afebrile after intervention $(3.1 \pm 2.7$ vs $3.8 \pm 2.9$ days, $\mathrm{p}=0.46$ ), and number of analgesic doses $(22.3 \pm 28.5$ vs $21.4 \pm 12, \mathrm{p}=0.9)$. Two patients in the VATS group required ongoing mechanical ventilation, and $3(16.6 \%)$ of the 18 patients treated with fibrinolysis required VATS therapy for definitive treatment. Although the clinical end points were not significantly different between groups in this study, a significant difference in overall hospital charges was observed between the two groups: $\$ 11,700 \pm 2900$ versus $\$ 7600 \pm 5400$ in the VATS versus fibrinolysis groups $(\mathrm{p}=0.02)$. Thus, it may be concluded that initial treatment with intrapleural tPA is a reasonable alternative to an initial VATS procedure and is associated with a lower total hospital cost.

\section{Safety}

Intrapleural tPA is generally well tolerated and associated with only minor adverse effects. ${ }^{31-35}$ Early case reports and case series noted mild discomfort, which was relieved by analgesics. ${ }^{31}$ One patient was reported to experience a drop in serum albumin level, with edema and ascites; however, this was most likely secondary to significant chest tube drainage. ${ }^{32}$

Sanguineous drainage without an associated drop in hematocrit, although reported, ${ }^{32}$ was believed to be secondary to initial chest tube placement rather than tPA treatment itself. An average drop in hemoglobin of $2.5 \mathrm{~g} / \mathrm{dl}$ was also observed in one case series, ${ }^{33}$ and minor bleeding into the pleural fluid has been described. ${ }^{34}$ Conversely, several reports noted no increased risk for bleeding. ${ }^{8}, 31,35,36$ Frank bleeding was described in a recent case report of a previously healthy 6-year-old boy treated with tPA $3 \mathrm{mg}$ in $30 \mathrm{ml}$ of normal saline $(0.1 \mathrm{mg} / \mathrm{kg})$ for pleural effusion. ${ }^{38}$ The patient developed clinical signs and symptoms of blood loss (tachypnea, weakness, and pallor); however, his neurologic status remained intact, and no other signs of bleeding were apparent. The patient's hemoglobin level dropped from $12.1 \mathrm{~g} / \mathrm{dl}$ to $7.6 \mathrm{~g} / \mathrm{dl}$, and an infusion of packed red blood cells was required. In light of this case report as well as conflicting safety outcomes reported in the literature, patients should be closely monitored for changes in hematocrit and hemoglobin and for clinical signs and symptoms of bleeding during therapy.

Few absolute contraindications for use of tPA exist. Potential contraindications include risk for bleeding, such as trauma, surgery, or recent major hemorrhage, or an allergy or hypersensitivity to tPA. ${ }^{12,}{ }^{14}$ Coagulopathy may also be considered to be a relative contraindication to tPA; however, therapy may be considered if the coagulopathy is mild and correctable. 5,33

\section{Remaining Questions and Future Directions}

Data for tPA in pediatrics are primarily retrospective, with only one randomized, prospective trial currently published. ${ }^{37}$ Nevertheless, evidence supports the use of tPA as a first-line option in pediatric patients for the management of complex pleural effusions. ${ }^{9,}{ }^{13}$ However, 
despite a growing body of literature and gains in clinical experience with this therapy, there are several questions that remain unanswered to date: timing of initiation of therapy, optimal dosing, frequency of doses, duration of therapy, dwell time, the need for positioning changes, and optimal therapeutic end points. In addition, although supported in the adult literature, the efficacy and safety of combination therapy with dornase alfa in pediatric patients has not yet been established.

Researchers evaluated the timing of administration of tPA and found that patients treated with early intervention, defined by intervention within 24 hours of diagnosis, experienced a shorter duration of chest tube placement. ${ }^{35}$ Although the clinical impact of this should be further studied, the ability to remove chest tubes earlier may improve patient comfort and patient mobility, and decrease analgesic dosing. Further, cost and duration of hospital stay may be lessened. Strong conclusions, however, cannot yet be made with respect to optimal timing due to data limited to retrospective evaluations.

The optimal dosing of tPA for pleural effusion in pediatric patients is also debatable based on the supporting literature. Due to heterogeneity among the studies, the optimal dose of tPA is unknown. Although some studies used fixed doses across age groups, others investigated a weight-based $(0.1 \mathrm{mg} / \mathrm{kg})$ approach. ${ }^{9},{ }^{31-37}$ The recent 2012 APSA publication suggests a fixed dose of $4 \mathrm{mg}$ based on the available prospective data. 9, 37 However, an alternative suggested weight-based dosing algorithm for tPA, constructed on extrapolation of the current data, is displayed in Figure 1. This approach has been used anecdotally with success at our institution but has not been validated or vigorously studied in a controlled manner. A prospective trial evaluating the safety and efficacy of this approach is warranted. Of note, the youngest patient reported in the literature to receive intrapleural tPA was 3 months, and the patient with the lowest weight reported was $5 \mathrm{~kg}$. Therefore, it is suggested that therapy be reserved for patients older than 3 months of age and above $5 \mathrm{~kg}$ in weight. The frequency of dosing, optimal duration of therapy, and dwell time are also yet undetermined due to variability in study design and published reports. However, the strongest evidence supports three doses given 24 hours apart, with a dwell time of 1 hour. ${ }^{37}$ Because the intrapleural pharmacokinetics of tPA are unknown, predicting the optimal parameters for therapy is difficult, and further studies are warranted.

Furthermore, the majority of the published data for intrapleural tPA in pediatric patients references a dilution of $0.1 \mathrm{mg} / \mathrm{ml}$; this dilution is also suggested in Figure 1 and has been used

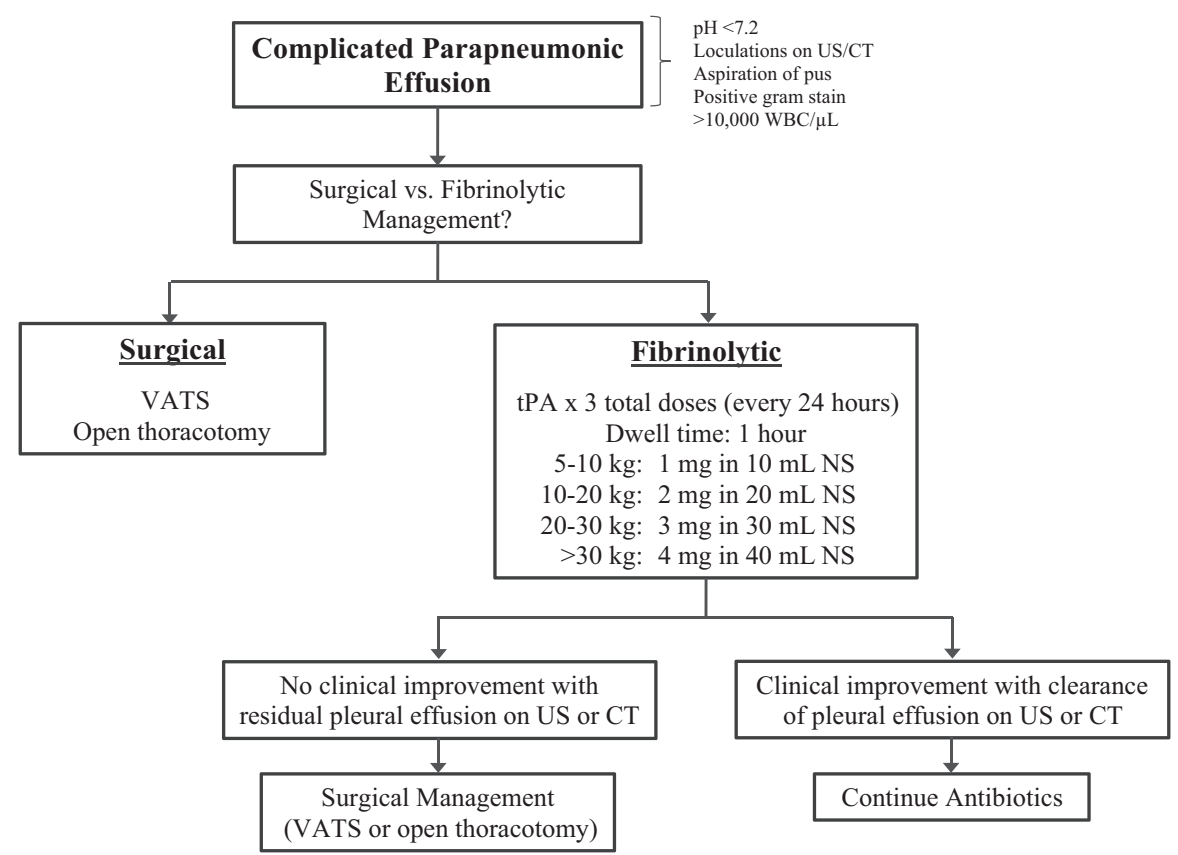

Figure 1. Proposed algorithm using weight-based dosing of tissue plasminogen activator for treatment of pleural effusion in pediatric patients. $\mathrm{tPA}=$ tissue plasminogen activator; US/CT $=$ ultrasound or computed tomography; VATS = video-assisted thoracoscopic surgery; $\mathrm{WBC}=$ white blood cell; NS = normal saline. 
with success at our institution. However, manufacturer recommendations for dilution, based on intravenous administration, note a final concentration of $1 \mathrm{mg} / \mathrm{ml}$, which may be diluted further immediately before administration to a concentration of $0.5 \mathrm{mg} / \mathrm{ml}$ with either dextrose $5 \%$ in water or normal saline. ${ }^{21}$ These solutions are stable at room temperature up to 8 hours. Alternative dilutions of tPA have been evaluated in vitro; however, the effects of these dilutions have not been evaluated clinically. In vitro data indicate that a tPA concentration of $0.01 \mathrm{mg} / \mathrm{ml}$ in normal saline is stable for up to 24 hours at room temperature. ${ }^{39}$ In that study, protein recovery was less than 50\%; but, biologic activity of the proteins recovered was preserved. Additional personal communication with Genentech (Caroline Smith, Pharm.D., Medical Communications Department, personal communication, March 20, 2011) suggests that tPA remains physically and chemically stable in normal saline at $0.2 \mathrm{mg} / \mathrm{ml}$. However, diluting tPA to concentrations less than $0.2 \mathrm{mg} / \mathrm{ml}$ in normal saline, less than $0.5 \mathrm{mg} / \mathrm{ml}$ in dextrose $5 \%$ in water, or diluting with other base solutions has reportedly resulted in protein precipitation. ${ }^{40}$ Although alternative dilutions are reported in the clinical data, most commonly $0.1 \mathrm{mg} / \mathrm{ml}$, further studies to confirm retained protein activity and stability at the concentration of $0.1 \mathrm{mg} /$ $\mathrm{ml}$ are warranted.

The optimal therapeutic end point of intrapleural tPA has not been elucidated, as the published literature is variable with respect to clinical end points. Several end points have been evaluated including change in pleural opacity seen on CXR, improved chest tube drainage, resolution of signs and symptoms, or resolution seen on ultrasound or CT. Determination of the optimal outcome should be a focus of future studies.

Furthermore, recent adult data support the use of intrapleural combination therapy, tPA plus dornase alfa, for initial management of parapneumonic effusion. However, the safety and efficacy of this approach has not been investigated in pediatric patients. Before consistently implementing dual intrapleural therapy in children, supporting data must become available, providing opportunities for future research.

\section{Conclusion}

Empyema and parapneumonic effusion are significant complications of pediatric pneumonia. Despite the overall decline in pneumonia rates in children, these conditions can significantly increase morbidity and mortality. Inadequate or delayed treatment may lead to progressive illness; therefore, identifying the optimal treatment in affected children is critical. Identifying patients who will not benefit from fibrinolytic treatment is difficult; however, surgical procedures can likely be reserved for patients who fail fibrinolysis. Although surgical treatments such as VATS and open thoracotomy are effective, they carry increased risk compared with chest tube drainage with or without fibrinolysis. Fibrinolytic agents such as tPA can help degrade loculations and dissolve fibrin deposits to facilitate drainage of purulent pleural fluid. Further large, randomized, prospective trials should be performed in order to answer some of the existing ongoing questions that remain regarding tPA therapy in pediatric patients with pleural effusion; however, sufficient data exist that support the use of tPA in these patients, with demonstrated efficacy and minimal safety concerns.

\section{References}

1. Hamm H, Light RW. Parapneumonic effusion and empyema. Eur Res J 1997;10:1150-6.

2. Light RW. Clinical practice. Pleural effusion. N Engl J Med 2002;346:1971-7.

3. Balfour-Lynn IM, Abrahamson E, Cohen G, et al. BTS guidelines for the management of pleural infection in children. Tho$\operatorname{rax}$ 2005;60(Suppl 1):il-21.

4. Jaffé A, Balfour-Lynn IM. Management of empyema in children. Pediatr Pulmonol 2005;40:148-56.

5. Colice GL, Curtis A, Deslauriers J, et al. Medical and surgical treatment of parapneumonic effusions: an evidence-based guideline. Chest 2000;18:1158-71.

6. Carter E, Waldhausen J, Zhang W, Hoffman L, Redding G. Management of children with empyema: pleural drainage is not always necessary. Pediatr Pulmonol 2010;45:475-80.

7. Grijalva CG, Nuorti JP, Zhu Y, Griffin MR. Increasing incidence of empyema complicating childhood communityacquired pneumonia in the United States. Clin Infect Dis 2010;50:805-13.

8. Stevens A, Tobias JD. Tissue plasminogen activator as adjunctive therapy of empyema in a child. J Intensive Care Med 2001;16:287-9.

9. Islam S, Calkins $\mathbf{C M}$, Goldin $\mathbf{A B}$, et al. The diagnosis and management of empyema in children: a comprehensive review from the APSA Outcomes and Clinical Trials Committee. J Pediatr Surg 2012;47:2101-10.

10. Sahn SA. Diagnosis and management of parapneumonic effusions and empyema. Clin Infect Dis 2007;45:1480-6.

11. Light RW. Parapneumonic effusions and empyema. Proc Am Thorac Soc 2006;3:75-80.

12. Hamblin SE, Furmanek DL. Intrapleural tissue plasminogen activator for the treatment of parapneumonic effusion. Pharmacotherapy 2010;30:855-62.

13. Bradley JS, Byington CL, Shah SS, et al. Executive summary: the management of community-acquired pneumonia in infants and children older than 3 months of age: clinical practice guidelines by the Pediatric Infectious Diseases Society and the Infectious Diseases Society of America. Clin Infect Dis 2011;53:617-30. 
14. Hawkins JA, Scaife ES, Hillman ND, Feola GP. Current treatment of pediatric empyema. Semin Thorac Cardiovasc Surg 2004;16:196-200.

15. Faber DL, Best LA, Orlovsky M, Lapidot M, Nir RR, Kremer R. Streptokinase fibrinolysis protocol: the advantages of a nonoperative treatment for stage II pediatric empyema patients. Isr Med Assoc J 2012;14:157-61.

16. Bouros D, Schiza S, Patsourakis G, Chalkiadakis G, Panagou $\mathrm{P}$, Siafakas NM. Intrapleural streptokinase versus urokinase in the treatment of complicated parapneumonic effusions: a prospective, double-blind study. Am J Respir Crit Care Med 1997; 155:291-5.

17. Stefanutti G, Ghirardo V, Barbato A, Gamba P. Evaluation of a pediatric protocol of intrapleural urokinase for pleural empyema: a prospective study. Surgery 2010;148:589-94.

18. Cochran JB, Tecklenburg FW, Turner RB. Intrapleural instillation of fibrinolytic agents for treatment of pleural empyema. Pediatr Crit Care Med 2003;4:39-43.

19. Bouros D, Tzouvelekis A, Antoniou KM, Heffner JE. Intrapleural fibrinolytic therapy for pleural infection. Pulm Pharmacol Ther 2007;20:616-26.

20. Abbott Laboratories. Abbokinase (urokinase) package insert. North Chicago, IL; 2002.

21. Genentech, Inc. Activase (alteplase) package insert. San Francisco, CA; 2011.

22. Genentech, Inc. Cathflo activase (alteplase) package insert. San Francisco, CA; 2010.

23. Micromedex. 2.0 [intranet database] 2013 Truven Health Analytics Inc. Alteplase, Recombinant. Available from http:// www.micromedexsolutions.com. Accessed July 8, 2013.

24. Gervais D, Levis D, Hahn P, Uppot RN, Arellano RS, Mueller PR. Adjunctive Intrapleural tissue plasminogen activator administered via chest tubes placed with imaging guidance: effectiveness and risk for hemorrhage. Radiology 2008;246: 956-63.

25. Levinson GM, Pennington DW. Intrapleural fibrinolytics combined with image-guided chest tube drainage for pleural infection. Mayo Clin Proc 2007;82:407-13.

26. Skeete DA, Rutherford EJ, Schlidt SA, Abrams JE, Parker LA, Rich PB. Intrapleural tissue plasminogen activator for complicated pleural effusions. J Trauma 2004;57:1178-83.

27. Thommi G, Shehan JC, Robison KL, Christensen M, Backemeyer LA, McLeay MT. A double blind randomized cross over trial comparing rate of decortication and efficacy of intrapleural instillation of alteplase vs. placebo in patients with empyemas and complicated parapneumonic effusions. Respir Med 2012;106:716-23.

28. Thommi G, Nair CK, Aronow WS, Shehan C, Meyers P, McLeay M. Efficacy and safety of intrapleural instillation of alteplase in the management of complicated pleural effusion or empyema. Am J Ther 2007;14:341-5.

29. Rahman NM, Maskell NA, West A, et al. Intrapleural use of tissue plasminogen activator and DNase in pleural infection. N Engl J Med 2011;365:518-26.

30. Walker CA, Shirk MB, Tschampel MM, Visconti JA. Intrapleural alteplase in a patient with complicated pleural effusion. Ann Pharmacother 2003;37:376-9.

31. Bishop NB, Pon S, Ushay HM, Greenwald BM. Alteplase in the treatment of complicated parapneumonic effusion: a case report. Pediatrics 2003;111:e188-90.

32. Ray TL, Berkenbosch JW, Russo P, Tobias JD. Tissue plasminogen activator as an adjuvant therapy for pleural empyema in pediatric patients. J Intensive Care Med 2004;19:44-50.

33. Feola GP, Shaw LC, Coburn L. Management of complicated parapneumonic effusions in children. Tech Vasc Interv Radiol 2003;6:197-204.

34. Wells RG, Havens PL. Intrapleural fibrinolysis for parapneumonic effusion and empyema in children. Radiology 2003;228:370-8.

35. Weinstein M, Restrepo R, Chait PG, Connolly B, Temple M, Macarthur C. Effectiveness and safety of tissue plasminogen activator in the management of complicated parapneumonic effusions. Pediatrics 2004;113(3 Pt 1):e182-5.

36. Gates RL, Hogan M, Weinstein S, Arca MJ. Drainage, fibrinolytics, or surgery: a comparison of treatment options in pediatric empyema. J Pediatr Surg 2004;39:1638-42.

37. St Peter SD, Tsao K, Spilde TL, et al. Thoracoscopic decortication vs. tube thoracostomy with fibrinolysis for empyema in children: a prospective, randomized trial. J Pediatr Surg 2009;44:106-11; discussion 111.

38. Hendaus MA, Abushahin A. Intrapleural hemorrhage due to alteplase use in a 6-year-old boy with pleural effusion. Int J Gen Med 2013;6:233-6.

39. Semba C, Weck S, Patapoff T. Alteplase: stability and bioactivity after dilution in normal saline solution. J Vasc Interv Radiol 2003;14:99-102.

40. Frazin BS, Magnuson D. Maximal dilution of activase. Am J Hosp Pharm 1990;47:1016. 